\title{
CATEGORY MEASURES ON BAIRE SPACES
}

\author{
J.M. AYERBE TOLEDANO
}

\begin{abstract}
The purpose of this paper is to give a necessary and sufficient condition to define a category measure on a Baire topological space. In the last section we give some examples of spaces in these conditions.
\end{abstract}

\section{Introduction}

A category measure in a topological space is a finite, countably additive measure $m$ defined on the class of the sets having the property of Baire and such that $m(E)=0$ if and only if $E$ is of first category.

The sets having the property of Baire in a space $X$ are those of the form $G+P$, where $G$ is open, $P$ is of first category, and " + "denotes symmetric difference. These sets constitute a $\sigma$-field of subsets of $X$.

In any topological space the open sets and the nowhere dense sets generate a field of subsets of $X$ called the complete basic ring of $X$. It consists of all sets of the form $G+N$, where $G$ is open and $N$ is nowhere dense. If $G$ is required to be regular open, that is, such that $G=G^{-^{\prime}-^{\prime}}$, then this representation of any element of the ring is unique. The regular open subsets of $X$ constitute a Boolean algebra $R(X)$ in which the Boolean sum, product and complement are defined respectively by $\left(G_{1} \cup G_{2}\right)^{\mathcal{\prime}^{\prime}-^{\prime}}, G_{1} \cap G_{2}$ and $G^{-^{\prime}}$, and the Boolean order relation by set inclusion. We shall call $R(X)$ the regular algebra of $X$. $R(X)$ is always complete. Moreover, any set having the property of Baire can be represented in the form $A=G+P$, where $G$ is a regular open set and $P$ is of first category. This representation is unique in any Baire space. In any topological space a set is called clopen if it is open and closed. The clopen subsets of $X$ constitute a Boolean algebra $B(X)$ in which the Boolean sum, product and complement are defined respectively by $G_{1} \cup G_{2}, G_{1} \cap G_{2}$ and $G^{\prime}$, and the Boolean order relation by set inclusion.

It is known that given a finite, complete measure space $(X, S, m)$ we can define a topology on $X$ with respect to which $m$ will be a category measure (see [7, chapter 22], [3], [8]).

In this paper we study the converse problem, that is, given a topological space, when can we define a finite and countably additive measure $m$ on the 
class of sets having the property of Baire with respect to which $m$ will be a category measure?

In this direction Oxtoby has shown [8, th. 1] that if $S$ is the union of all open sets of first category in a topological space $X$, then $X$ admits a category measure if and only if $X-S^{-}$is non-empty and $R\left(X-S^{-}\right)$admits a finite, strictly positive and countably additive measure. In particular, a Baire space admits a category measure if and only if its regular algebra admits a finite, strictly positive and countably additive measure. Moreover, because $X-S^{-}$is a Baire space, it follows that in the study of this subject, we may confine our attention to Baire space without essential loss of generality.

This theorem shows that the problem of defining a category measure on a topological Baire space can be reduced to the problem of defining a finite, strictly positive and countably additive measure on a Boolean algebra. The general problem of the existence of measures on Boolean algebras has been abundantly studied (see [4], [5] and [6]). In [5, Addendum] Ryll-Nardzewski gives a necessary and sufficient condition. The concept of intersection number has been defined by Kelley for Boolean algebras, and has been translated to topological spaces in [1, Def. 0.3]. We shall use the terminology of this paper.

\section{A necessary and sufficient condition}

Definition 2.1. A topological space $(X, T)$ has the property $\left({ }^{* * *}\right)$ if and only if there exists a decomposition of $T^{*}=T-\{9\}$ into a sequence $\left\{T_{n}: n<w\right\}$ such that:

1) $k\left(T_{n}\right)>0$ for each $n<w$.

2) If $\left\{A_{m}: m<w\right\}$ is a increasing sequence of open sets such that $\bigcup_{m=1}^{\infty} A_{m} \in T_{n}$, there is a natural number $m$ with $A_{m} \in T_{n}$.

3) If $V, W$ are open sets, $V \sim W$ (i.e., $V+W$ is a first category set) and $V \in T_{n}$, then $W \in T_{n}$.

Theorem 2.2. Let $X$ be a topological Baire space. Then $X$ has the property $\left(^{* * *}\right)$ if and only if $X$ admits a category measure.

Proof: Suppose that $X$ has the property $\left({ }^{* *}\right)$. Then there exists a decomposition of $T^{*}$ in the conditions of Definition 2.1 .

Let $T_{n}=\left\{V_{i}^{(n)}: i \in I^{(n)}\right\}$ for each $n<w$.

For each $i \in I^{(n)}$ let $H_{i}^{(n)}=V_{i}^{(n)--^{\prime}}$. Then $H_{i}^{(n)}$ is a regular open set such that $V_{i}^{(n)} \subseteq H_{i}^{(n)}$ and are equivalents.

Let $T_{n}^{\prime}=\left\{H_{i}^{(n)}: i \in I^{(n)}\right\}$. Then:

a) $R(X)^{*}=\bigcup_{n=1}^{\infty} T_{n}^{t}$

b) $K\left(T_{n}^{\prime}\right) \geq K\left(T_{n}\right)$ for each $n<w$

It follows immediately from $V_{i}^{(n)} \subseteq H_{i}^{(n)}$ for each $n<w, i \in I^{(n)}$. 
c) Let $\left\{A_{m}: m<w\right\}$ be an increasing sequence in $R(X)$ such that $\bigcup_{m=1}^{\infty} A_{m} \in T_{n}^{\prime}$. We shall show that there is a natural number $m$ such that $A_{m} \in T_{n}^{\prime}$.

Indeed: Since $\bigcup_{m=1}^{\infty} A_{m} \in T_{n}^{\prime}$, it follows that $\left(\bigcup_{m=1}^{\infty} A_{m}\right)^{-^{\prime}-^{\prime}} \in T_{n}^{\prime}$, and therefore there is $V \in T_{n}$ such that $V^{-^{\prime}-^{\prime}}=\left(\bigcup_{m=1}^{\infty} A_{m}\right)^{-^{\prime}-^{\prime}}$. Thus $V \in T_{n}$ and $V \sim \bigcup_{m=1}^{\infty} A_{m}$, and hence $\bigcup_{m=1}^{\infty} A_{m} \in T_{n}$.

It follows that there is a natural number $m$ such that $A_{m} \in T_{n}$. Thus $A^{-^{\prime}-^{\prime}}=A_{m} \in T_{n}^{\prime}$.

Therefore we obtain that the Boolean algebra $R(X)$ satisfes the condition of Ryll-Nardzewski to admit a finite, strictly positive and countably additive measure. Thus $(X, T)$ admits a category measure by $[8, T h .1]$.

Conversely, suppose that $X$ admits a category measure. Then $R(X)$ admits a finite, strictly positive and countably additive measure. Therefore there exists a decomposition of $R(X)^{*}$ into a sequence $\left\{T_{n}^{*}: n<w\right\}$ in the conditions of Ryll-Nardzewski's theorem.

We consider now the following decomposition of $T^{*}$ :

Let $H \in T^{*}$ and $G \in R(X)$ such that $G \sim H$. Suppose that $G \in T_{n}^{*}$. Then $H \in T_{n}$. It is easy to see that every open set belongs to a unique $T_{n}$.

Thus the obtained sequence $\left\{T_{n}: n<w\right\}$ satisfies the following properties:

a) $T^{*}=\bigcup_{n=1}^{\infty} T_{n}$

b) By construction, if $U, V \in T^{*}, U \sim V$ and $U \in T_{n}$, then $V \in T_{n}$.

c) $K\left(T_{n}\right)>0$ for each $n<w$.

Indeed: We write for $n$ fixed $T_{n}^{*}=\left\{G_{l}: l \in L\right\}$ and let $T_{n}=\left\{\left[G_{l}\right]: l \in L\right\}$, where for each $l \in L\left[G_{l}\right]=\left\{V_{i}^{l}: i \in I^{l}\right\}, V_{i}^{l} \in T^{*}, V_{i}^{l} \sim G_{l}$ for each $i \in I^{l}$.

We consider the decomposition of $R(X)^{*}$ by the sequence $\left\{T_{n}^{* *}: n<w\right\}$, wherc $T_{n}^{* *}=\left\{\left[G_{i}\right]^{*}: i \in L\right\},\left[G_{i}\right]^{*}=\left\{G_{i}: i \in I^{i}\right\}$.

Obviously $k\left(T_{n}^{* *}\right)>0$ and $\left|T_{n}\right|=\left|T_{n}^{* *}\right|$. Moreover, given a finite set of indices $J=\left\{j_{1}, \ldots, j_{n}\right\}$ corresponding in $T_{n}$ to the open sets $V_{j_{1}}, \ldots, V_{j_{n}}$ and in $T_{n}^{* *}$ to the regular open sets $G_{j_{1}}, \ldots G_{3_{n}}$ and such that $V_{j_{i}} \sim G_{j_{i}}$ for each $i=1, \ldots, n$, it is easy to prove that $\bigcap_{\substack{i \in J^{\prime} \\ J^{\prime} \subseteq J}} V_{j_{i}} \neq \emptyset$ if and only if $\bigcap_{\substack{i \in J^{\prime} \\ J^{\prime} \subset J}} G_{j_{i}} \neq \emptyset$, because if these sets are non empty, then they are equivalent second category sets.

It follows that $\mathrm{cal}_{T_{n}}(J)=\operatorname{cal}_{T_{n} \cdot}(J)$ for each $J$ finite, and hence $k\left(T_{n}\right)=$ $k\left(T_{n}^{* *}\right)>0$.

d) Let $\left\{A_{m}: m<w\right\}$ be an increasing sequence of open sets such that $\bigcup_{m=1}^{\infty} A_{m} \in T_{n}$. For each natural number $m$ we consider the regular open sets $A_{m}^{--^{\prime}}$ (equivalent to $A_{m}$ ). Thus $\bigcup_{m=1}^{\infty} A_{m}^{-^{\prime}--^{\prime}} \sim \bigcup_{m=1}^{\infty} A_{m}$, and since $\bigcup_{m=1}^{\infty} A_{m}^{-^{\prime}-^{\prime}} \sim \vee_{m=1}^{\infty} A_{m}^{-^{\prime}-^{\prime}}$, it follows that $\bigcup_{m=1}^{\infty} A_{m}^{-^{\prime}-^{\prime}} \sim \vee_{m=1}^{\infty} A_{m}^{-^{\prime}-^{\prime}} \sim$ $\bigcup_{m=1}^{\infty} A_{m} \in T_{n}$. Hence $\vee_{m=1}^{\infty} A_{m}^{-^{\prime}-^{\prime}} \in T_{n}$.

Since $V_{m=1}^{\infty} A_{m}^{-\prime^{\prime}} \in T_{n}$, by construction there exists a regular open set $V$ such that $V \sim \vee_{m=1}^{\infty} A_{m}^{-^{\prime}-^{\prime}}$ and $V \in T_{\pi}^{*}$. It is easy to check that $V=\vee_{m=1}^{\infty} A_{m}^{-^{\prime}-^{\prime}}$, 
and therefore $V_{m=1}^{\infty} A_{m}^{--^{\prime}} \in T_{n}^{*}$.

Thus $\vee_{m=1}^{\infty} A_{m}^{-^{\prime}-^{\prime}} \in T_{n}^{*}$ and $\left\{A_{m}^{-^{\prime}-^{\prime}}: m<w\right\}$ is an increasing sequence of regular open sets. It follows that there exists a natural number $m$ such that $A_{m}^{--^{\prime}} \in T_{n}^{* *}$, and since $A_{m} \sim A_{m}^{--^{\prime}}$, we obtain that $A_{m} \in T_{n}$. Thus the proof is finished.

Remark 2.3. Property $\left({ }^{* * *}\right)$ implies property $\left({ }^{* *}\right)$ (see [1, Def. 0.4]). The converse is not true. Indeed: Let $\mathbb{R}$ be the set of real numbers with the Euelidean topology $T$. This topology is second countable, and therefore there is a countable base $\mathcal{B}=\left\{B_{n}: n<w\right\}$ of $T$. For each $n<w$ let $T_{n}=\{V \in T$ : $\left.B_{n} \subseteq V\right\}$. Then for each $n<w, k\left(T_{n}\right)=1$. Thus $\mathbb{R}$ has property $\left({ }^{* *}\right)$.

If $\mathbb{R}$ had property ( ${ }^{* * *}$ ), then the topological space $(\mathbb{R}, T)$ would admit a category measure by theorem 2.2. This is not true because in $\mathbb{R}$ there are sets of first category that are not nowhere dense (for example $Q$ ), contradicting $[8$, Th. 3], Thus $(\mathbf{R}, T)$ has not property $\left({ }^{* *}\right)$.

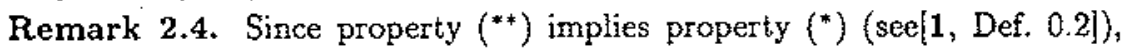
and property $\left({ }^{*}\right)$ implies $C C C$ (countable chain condition), it follows that the following diagram holds

$$
\left({ }^{* *}\right) \Longrightarrow\left({ }^{* *}\right) \Longrightarrow\left(^{*}\right) \Longrightarrow C C C
$$

The converse of these implications is not true in general. Nevertheless, we have proved (see [2, note 7$]$ ) that if every open set contains a minimal open set then $C C C$ implies that $X$ admits a category measure, and therefore

$$
\left({ }^{* *}\right) \Longleftrightarrow\left({ }^{* *}\right) \Longleftrightarrow\left({ }^{*}\right) \Longleftrightarrow C C C
$$

Remark 2.5. A concrete example of a topological space with the property $\left({ }^{* *}\right)$ is the following:

Let 1 be the set of real numbers, $M$ the $\sigma$-algebra of Lebesgue measurable sets, $\mathcal{N}$ the class of Lebesgue nullsets, and

$$
T d=\{\emptyset(A)-N: A \in \mathcal{M}, N \in \mathcal{N}\}
$$

where $\theta$ is the Lebesgue lower density (see [7, page 16]).

It is proved in [7, page 88-90] that $T_{d}$ is a topology of Baire that admits a category measure. Thus, it follows from theorem 2.2 that $\left(\mathbb{R}, T_{d}\right)$ has the property $\left({ }^{* * *}\right)$. This topology has been abundantly studied in [9].

\section{Examples}

Example 3.1. Let $(X, T)$ be a Baire topological space that satisfies:

a) $X$ is second countable.

b) $X$ is countably compact. 
c) Each open set of the topology is a closed set.

Then $(X, T)$ has property (***) and, therefore it admits a category measure.

Proof: Let $\mathcal{B}=\left\{G_{n}: n<w\right\}$ a countable base of $T$, and let $T_{n}=\{V \in T$ : $\left.G_{n} \subseteq V\right\}$ for each $n<w$. Then the sequence $\left\{T_{n}: n<w\right\}$ satisfies property $\left({ }^{* * *}\right)$.

A less trivial example is the following:

Example 3.2. Let $(X, T)$ be a topological space that satisfies:

a) $X$ is second countable.

b) $X$ is Hausdorff compact.

c) $X$ is 0-dimensional.

d) The closure of every open set in $X$ is open.

e) If $\left\{A_{m}: m<w\right\}$ is an increasing sequence of open sets, then there is a natural number $m$ such that $A_{m} \sim \bigcup_{m=1}^{\infty} A_{m}$.

Then $(X, T)$ satisfies property $\left({ }^{* *}\right)$ and, therefore it admits a category measure.

Proof: Let $B(X)$ and $R(X)$ the clopen algebra and the regular algebra of $X$, respectively.

By c) and d) $R(X)=B(X)$ (see [8, Th. 10 and Corollary]).

Moreover, from a), b) and c) it follows that $B(X)$ is countable.

Let $B(X)=\left\{G_{n}: n<w\right\}$ and $T_{n}=\left\{V \in T: V \sim G_{n}\right\}$ for each $n<w$. Since $X$ is a Baire topological space, for each open set $V$ there is a unique clopen set $G_{n}$ such that $V \sim G_{n}$. Thus $T_{n} \cap T_{m}=$ for each pair of natural numbers $n, m, n \neq m$. Moreover the sequence $\{T n: n<w\}$ satisfies property $(* *)$.

We shall show some examples of topological spaces in this conditions, tutilizing terminology and properties of [3].

Proposition 3.1. Let $(X, T)$ be a Boolean $\sigma$-space second countable. Let $B(X)$ be the family of clopen sets of $X$. Suppose that $B(X)$ satisfies the finite chain condition. Then $(X, T)$ satisfies property $\left({ }^{* * *}\right)$ and, therefore it admits a category measure.

Proof: We shall show that $(X, T)$ is in the conditions of example 3.2. Since $X$ is a Boolean $\sigma$-space second countable, $X$ satisfies a), b) and c). By a) and c) it follows that any open set is a Baire open set. Thus we obtain d). To check e), let $\left\{A_{m}: m<w\right\}$ be an increasing sequence of open sets. We can suppose that the sequence is strictly increasing without loss of generality.

Then $\left\{A_{m}^{-}: m<w\right\}$ is an increasing sequence of clopen sets (again we can suppose that the sequence is strictly increasing), and therefore finite (following 
an argument as in $[3, \S 14$, lemma 2$])$. Thus there is a natural number $m_{0}$ such that $A_{m_{0}}^{-}=\bigcup_{m=1}^{\infty} A_{m}^{-}$.

But $A_{m_{0}} \sim A_{m_{0}}^{-}$and $A_{m_{0}}^{-}=\bigcup_{m=1}^{\infty} A_{m_{0}}^{-} \sim \bigcup_{m=1}^{\infty} A_{m}$. Hence $A_{m_{0}} \sim$ $\bigcup_{m=1}^{\infty} A_{m}$ and we obtain e).

Thus $(X, T)$ is in the conditions of example 3.2 and therefore satisfies $\left(^{* * *}\right)$.

Some examples of topological spaces in the conditions of proposition 3.1 follow immediately from the theory of Boolean algebras. Thus we have

Proposition 3.2. Let $\mathcal{A}$ be a countable Boolean $\sigma$-algebra with the finite chain condition. Then the Stone's space $X$ of $\mathcal{A}$ satisfies property $\left({ }^{* * *}\right)$ and, therefore it admits a category measure.

Proof: The Stone's space $X$ of $\mathcal{A}$ is a Hausdorf compact and 0-dimensional space, and its clopen algebra $B(X)$ is isomorphic to $\mathcal{A}$ by the classical Stone's representation theorem. It follows that $B(X)$ is a $\sigma$-algebra, and therefore $X$ is a $\sigma$-space (see $[3, \S 22$, Th. 12]). Since $A$ is countable we obtain that $X$ is second countable and, therefore, $B(X)$ is countable. Thus $B(X)$ is complete and hence the closure of every open set in $X$ is open (see [8, Th. 10 and Corollary]). Finally, since $B(X)$ satisfies the finite chain condition, we obtain the condition e) following an argument as in the proof of the proposition 3.1.

In this paper we have always used the Ryll-Nardzewski's condition in order to a Boolean algebra admits a finite, strictly positive and countably additive measure. An analogue condition has been shown by Kelley in [5, Th. 4 and 9$]$. We shall use this condition in the following

Example 3.3. Let $(X, T)$ a topological space that satisfies:

a) $X$ is second countable.

b) $X$ is a Hausdorf compact.

c) $X$ is 0-dimensional.

d) The closure of every open set in $X$ is open.

e) Every set of first categary in $X$ is nowhere dense.

Then $(X, T)$ admits a category measure and, therefore it satisfies property $(* * *)$.

Proof: An argurnent as in the example 3.2 shows that $R(X)=B(X)$ and that $B(X)$ is countable.

From $[5$, Th. 8$]$ it follows that $B(X)$ is weakly countably distributive and, therefore $B(X)$ admits a finite, strictly positive and countably additive measure. Thus $(X, T)$ admits a category measure.

Some examples of topological spaces in the conditions of example 3.3 are given in the following 
Proposition 3.3. Let $\mathcal{A}$ be a weakly countably distributive and countable Boolean $\sigma$-algebra. Then the Stone's space $X$ of $\mathcal{A}$ is in the conditions of example 3.9 and therefore it admits a category measure and it satisfies property $\left({ }^{* * *}\right)$.

Proof: We argue as in proposition 3.2 .

\section{References}

1. S. Argyros, On compact spaces without strictly positive measure, Pacific J. Math. 105 (1983), 257-272.

2. J.M. AYERBE, Algunos espacios topologicos que admiten una medida categoria, Collectanea Math. XXXV, Fasc. 3 (1984), 221-231.

3. P.R. Halmos, "Lectures on Boolean algebras," Springer-Verlag, 1974.

4. A. Horn, A. TARsKI, Measures in Boolean algebras, Trans. Amer. Math. Soc. $64(1948), 467-497$.

5. J.L. Kelley, Measures on Boolean algebras, Pacific J. Math 9 (1959), 1165-1177.

6. D. MAhaRAM, An algebraic characterization of measure algebras, Annals of Math 48 (1947), 154-167.

7. J.C. Oxтову, "Measure and Category," Springer-Verlag, 1971.

8. I.C. Oxтовy, Spaces that admit a category measure, J. Reine Angew. Math 205 (1961), 156-170.

9. F.D. TALl, The density topology, Pacific J. Math. 62 (1976), 275-284.

Departamento de Análisis Matemático

Facultad de Matemáticas

Apartado de Correos 1160

41080-Sevilla

SPAIN

Rebut el 25 d'Octubre de 1989 